\title{
Stresslets Induced by Active Swimmers
}

\author{
Eric Lauga ${ }^{1, *}$ and Sébastien Michelin ${ }^{2, \dagger}$ \\ ${ }^{1}$ Department of Applied Mathematics and Theoretical Physics, University of Cambridge, Cambridge CB3 OWA, United Kingdom \\ ${ }^{2}$ LadHyX-Département de Mécanique, Ecole Polytechnique-CNRS, 91128 Palaiseau, France
}

(Received 6 June 2016; published 30 September 2016)

\begin{abstract}
Active particles disturb the fluid around them as force dipoles, or stresslets, which govern their collective dynamics. Unlike swimming speeds, the stresslets of active particles are rarely determined due to the lack of a suitable theoretical framework for arbitrary geometry. We propose a general method, based on the reciprocal theorem of Stokes flows, to compute stresslets as integrals of the velocities on the particle's surface, which we illustrate for spheroidal chemically active particles. Our method will allow tuning the stresslet of artificial swimmers and tailoring their collective motion in complex environments.
\end{abstract}

DOI: 10.1103/PhysRevLett.117.148001

The study of swimming microorganisms could be hailed as the biophysics "poster child" due to the ability of classical physics to provide robust quantitative predictions $[1,2]$. Mathematical theories developed from first principles have been able to quantitatively capture the locomotion of bacteria [3], spermatozoa [4], and algae [5], as well as their collective dynamics [6] and their interactions with complex chemical environments [7]. In addition, self-propelling cells and artificial active particles $[8,9]$ have provided the soft matter community with model systems to discover new physics $[10,11]$.

The primary quantity of interest for a swimming body, and what most theory work focuses on, is its swimming speed $U$. A wealth of experimental data exists for a large variety of biological cells [12]. Mathematical methods have been developed to predict swimming speeds, in particular, resistiveforce [13] and slender-body theory [14]. These solve for the force distribution along an organism by taking advantage of the linearity of the Stokes equations for the fluid flow to determine the swimming kinematics without requiring a full computation of the flow. With its swimming speed known, a swimmer is then seen to display long-time effective diffusion at a rate $D \sim U^{2} \tau$, where the time scale $\tau$ is the relevant one for loss of orientation, be it thermal noise or cell tumbling [15].

Beyond the swimming speed, an equally important characteristic of a self-propelled body is its stresslet. Since cells and active particles swim without applying net forces to the surrounding fluid, the flows they induce have the symmetry of a force dipole and decay spatially as $\sim 1 / r^{2}$. Formally, the velocity field in the laboratory frame at a location $\mathbf{x}$ away from a swimmer can generically be written in the far field as $\mathbf{u}=-3(\mathbf{x} \cdot \mathbf{S} \cdot \mathbf{x}) \mathbf{x} / 8 \pi \mu r^{5}$, where $r=|\mathbf{x}|$ and $\mathbf{S}$ is the trace-free second-rank stresslet tensor which is symmetric when the swimmer does not apply any net moment [16]. For axisymmetric swimming along a direction $\mathbf{e}$, one then obtains $\mathbf{S}=S\left(\mathbf{e e}-\frac{1}{3} \mathbf{I}\right)$, and the sign of $S$ allows one to distinguish between two types of swimmers: pusher cells with $S<0$ are pushed from behind and include most flagellated bacteria; in contrast, puller cells with $S>0$ are pulled forward, e.g., the biflagellated algae Chlamydomonas.

The stresslets of self-propelling cells and active particles have been the subject of much less attention than their swimming speeds, but they are no less important. The magnitudes and signs of stresslets control pattern formation and interactions in populations of cells [17], dictate which type of swimmer suspension is unstable and displays nonlinear fluctuations [18], and govern the physics of collective locomotion $[19,20]$. The stresslet also controls the interactions of active organisms with their environment $[21,22]$, enhanced transport through biological fluids $[23,24]$, and the rheology of active fluids [25].

If the stresslet of active swimmers is so important, why do so few studies attempt to determine its value? The difficulty lies in the fact that, unlike the swimming speed which is purely a kinematic quantity, the stresslet includes information about both kinematics and dynamics as it is formally given by an integral on the surface of the swimmer of both instantaneous surface velocities and surface stresses [16]. Solving for both velocities and surface stresses can be done numerically using the boundary element method [26], but typically not analytically. An alternative method consists of measuring, or computing, the flow far from the swimmer and fitting it to the expected stresslet, but so far this has been done only with the bacterium E. coli [27] and requires an experimental apparatus able to distinguish the far-field flow from measurement noise.

In this Letter, we propose a theoretical method to compute the stresslet induced by active swimmers. Twenty years ago, Stone and Samuel derived an integral theorem to determine the swimming speed of any swimmer using an auxiliary problem of rigid-body motion [28]. This result relies on the Lorentz reciprocal theorem, which has proved popular in the hydrodynamics community to compute Marangoni, inertial, or viscoelastic effects on the motion of particles, drops, and bubbles [29-31], and even the flux of boundary-driven 
channel flows [32]. We show that a similar approach may be undertaken to determine the value of the stresslet for active particles of arbitrary shape. We derive a new integral theorem, involving an auxiliary problem of a passive rigid particle in a linear flow, allowing the determination of the full stresslet tensor. After validating it for the classical problems of swimming of a sphere (squirming) and locomotion of an active rod, we show that the theorem allows one to determine exactly, for the first time, the stresslet induced by ellipsoidal swimmers of any aspect ratio. We apply our results to phoretic particles and discover how the pusher-puller transition depends on the geometry of the particle.

In seminal work, Batchelor [16] showed that the contribution of an active particle of surface $\partial V$ to the bulk stress, i.e., the so-called stresslet tensor $\mathbf{S}$, is given by

$$
\begin{aligned}
S_{i j}= & \int_{\partial V}\left[\frac{1}{2}\left(x_{j} \sigma_{i k} n_{k}+x_{i} \sigma_{j k} n_{k}\right)\right. \\
& \left.-\frac{1}{3}\left(x_{k} \sigma_{k l} n_{l}\right) \delta_{i j}-\mu\left(u_{i} n_{j}+u_{j} n_{i}\right)\right] d A .
\end{aligned}
$$

For active particles or cells prescribing a relative surface velocity $\mathbf{u}^{s}$ (or swimming gait), the second part of this integral can be directly evaluated (its value does not depend on the swimming velocity). In contrast, the first part involves the surface traction, $\boldsymbol{\sigma} \cdot \mathbf{n}$, which in general can only be obtained by solving for the flow everywhere. In order to calculate this first part of the stresslet integral, we use the reciprocal theorem of Stokes flow written as [33]

$$
\int_{\partial V} u_{i} \bar{\sigma}_{i j} n_{j} d A=\int_{\partial V} \bar{u}_{i} \sigma_{i j} n_{j} d A
$$

where we choose the dual flow field $(\overline{\mathbf{u}}, \overline{\boldsymbol{\sigma}})$, a solution of Stokes's equations that decays at infinity, to satisfy $\overline{\mathbf{u}}=$ $\mathbf{E} \cdot \mathbf{x}$ on the particle's boundary, where $\mathbf{E}$ is a constant, symmetric, and traceless second-order tensor, and the origin of $\mathbf{x}$ is chosen so that the particle is force and torque free. The solution $(\overline{\mathbf{u}}, \overline{\boldsymbol{\sigma}})$ is thus the instantaneous perturbation flow induced by the presence of the same active particle when stationary in a linear flow field; i.e., $\mathbf{u}=-\mathbf{E} \cdot \mathbf{x}+\overline{\mathbf{u}}$. The associated stress field can be formally written as $\overline{\boldsymbol{\sigma}}(\mathbf{x}) \equiv \mu \boldsymbol{\Sigma}(\mathbf{x}): \mathbf{E}$, where $\boldsymbol{\Sigma}$ is a dimensionless fourth-order tensor symmetric with respect to the first two and last two indices (due to the symmetries of $\overline{\boldsymbol{\sigma}}$ and $\mathbf{E}$ ).

After changing indices, the left-hand side of Eq. (2) becomes

$$
\int_{\partial V} u_{i} \bar{\sigma}_{i j} n_{j} d A=\mu\left(\int_{\partial V} n_{l} u_{k}^{s} \Sigma_{k l i j} d A\right) E_{i j}
$$

whereas the right-hand side is

$$
\int_{\partial V} \bar{u}_{i} \sigma_{i j} n_{j} d A=\left(\int_{\partial V} \frac{1}{2}\left(x_{j} \sigma_{i k} n_{k}+x_{i} \sigma_{j k} n_{k}\right) d A\right) E_{i j},
$$

where the term in parentheses has been replaced by its symmetric part since $\mathbf{E}$ is symmetric. Equating Eqs. (3) and (4), for any trace-free symmetric tensor $\mathbf{E}$, we obtain

$$
\int_{\partial V} \frac{1}{2}\left(x_{j} \sigma_{i k} n_{k}+x_{i} \sigma_{j k} n_{k}\right) d A=\mu \int_{\partial V} n_{l} u_{k}^{s} \Sigma_{k l i j} d A,
$$

up to an isotropic second-order tensor. The trace-free portion of this result is given by

$$
\begin{gathered}
\int_{\partial V}\left[\frac{1}{2}\left(x_{j} \sigma_{i k} n_{k}+x_{i} \sigma_{j k} n_{k}\right)-\frac{1}{3}\left(x_{k} \sigma_{k l} n_{l}\right) \delta_{i j}\right] d A \\
=\mu \int_{\partial V} n_{l} u_{k}^{s}\left(\Sigma_{k l i j}-\frac{1}{3} \Sigma_{k l m m} \delta_{i j}\right) d A .
\end{gathered}
$$

Combining Eqs (1) and (6), we finally obtain the stresslet tensor $\mathbf{S}$ as

$\frac{S_{i j}}{\mu}=\int_{\partial V} n_{l} u_{k}^{s}\left(\Sigma_{k l i j}-\frac{\delta_{i j}}{3} \Sigma_{k l m m}-\delta_{i k} \delta_{j l}-\delta_{i l} \delta_{j k}\right) d A$.

The result in Eq. (7) is an explicit integral of the prescribed, or measured, surface velocity $\mathbf{u}^{s}$, and does not depend on the swimming velocity of the particle — similarly to Eq. (1). Provided $\boldsymbol{\Sigma}$ can be computed once and for all for the same geometry (either analytically or numerically), this result allows one to directly compute the stresslet generated by the active particle or cell for any surface velocity and without actually solving the associated flow problem.

This integral formulation can first be used to recover classical results, starting with the stresslet induced by a squirming sphere [34]. The dual flow field $\overline{\mathbf{u}}$ for a sphere of radius $a$ in a linear flow is a classical solution given by [33]

$$
\begin{gathered}
\overline{\mathbf{u}}=a^{5} \frac{\mathbf{E} \cdot \mathbf{x}}{r^{5}}+\frac{5(\mathbf{x} \cdot \mathbf{E} \cdot \mathbf{x}) \mathbf{x}}{2}\left(\frac{a^{3}}{r^{5}}-\frac{a^{5}}{r^{7}}\right), \\
\bar{p}=5 a^{3} \mu \frac{\mathbf{x} \cdot \mathbf{E} \cdot \mathbf{x}}{r^{5}} .
\end{gathered}
$$

From this, the tensor $\overline{\boldsymbol{\sigma}}$ and thus $\boldsymbol{\Sigma}$ may be easily evaluated [35]. Using Eq. (7), the stresslet is obtained as

$\mathbf{S}=\mu \int_{\partial V}\left(5 n_{i} n_{j} n_{k}-\frac{5}{2}\left(n_{j} \delta_{i k}+n_{i} \delta_{j k}\right)+\delta_{i j} n_{k}\right) u_{k}^{s} d A$.

For an axisymmetric squirming sphere [34], the prescribed slip velocity is purely tangential, $\mathbf{u}^{s}=u^{s}(\zeta) \mathbf{e}_{\theta}(\zeta=\cos \theta$ in spherical polar coordinates). In that case, the stresslet simplifies to

$$
\mathbf{S}=-\frac{5 \mu}{2} \int_{\partial V}\left(\mathbf{n} \mathbf{u}^{s}+\mathbf{u}^{s} \mathbf{n}\right) d A,
$$

and finally, 


$$
\mathbf{S}=15 \pi \mu a^{2}\left(\mathbf{e}_{z} \mathbf{e}_{z}-\frac{1}{3} \mathbf{I}\right) \int_{-1}^{1} u^{s}(\zeta) \zeta \sqrt{1-\zeta^{2}} d \zeta .
$$

This result is equivalent to decomposing the slip velocity onto the canonical squirming modes, with the second mode providing the intensity of the stresslet $[34,37,38]$.

Another classical model is the active rod. A rod of length $L$ and unit direction vector $\mathbf{p}$ imposes an axisymmetric slip velocity $\mathbf{u}^{s}=\alpha(s) \mathbf{p}$ in its reference frame, with $-L / 2 \leq$ $s \leq L / 2$ the arc length measured along the rod. To determine the stresslet, the force distribution acting on a rigid rod in a linear flow $\mathbf{u}=-\mathbf{E} \cdot \mathbf{x}$ must be computed. The integral to calculate in Eq. (7) is

$$
\mu \int_{\partial V} n_{l} u_{k} \Sigma_{k l i j} d A=\int_{L} u_{k} \int_{\partial V_{R}} \mu n_{l} \Sigma_{l k i j} d A
$$

where $n_{l} \Sigma_{l k i j}$ is obtained through the force per unit length acting on the rigid rod as

$$
\bar{f}_{k}=\left(\int_{\partial V_{R}} \mu n_{l} \Sigma_{k l i j} d A\right) E_{i j} .
$$

The force density $\overline{\mathbf{f}}$ can be obtained using resistive-force theory [2,13] (with $\mathbf{x}=s \mathbf{p}$ ),

$$
\overline{\mathbf{f}}(s, t)=s \zeta_{\perp}\left(\frac{\mathbf{p p}}{2}-\mathbf{I}\right) \cdot \mathbf{E} \cdot \mathbf{p},
$$

and thus,

$$
\int_{\partial V_{R}} \mu n_{l} \Sigma_{k l i j} d A=s \zeta_{\perp}\left(\frac{p_{i} p_{k}}{2}-\delta_{i k}\right) p_{j},
$$

where $\zeta_{\perp}$ is the perpendicular drag coefficient for the rod $[2,13]$. Using these results, Eq. (7) becomes finally

$$
\mathbf{S}=-\left(\frac{1}{2} \zeta_{\perp} U_{0} \int_{L} s \alpha(s) d s\right)\left(\mathbf{p p}-\frac{1}{3} \mathbf{I}\right),
$$

which is identical to the result of a direct calculation [35].

The power of the integral method in Eq. (7) may be demonstrated on problems where a direct calculation of $\mathbf{S}$ is not tractable analytically. Motivated by recent work on phoretic swimmers, we illustrate this for an axisymmetric active spheroidal particle (or swimmer) of axis $\mathbf{e}_{z}$ and semiaxes $a$ and $b$. In this case, the flow field can still be computed as a superposition of spheroidal harmonics [39], but a direct calculation of the tensor $\mathbf{S}$ from a projection of $\mathbf{u}^{s}$ on the relevant harmonics is much more difficult. In contrast, the integral formulation allows one to determine $\mathbf{S}$ exactly and explicitly, for an arbitrary $\mathbf{u}^{s}$.

Focusing on an axisymmetric distribution of slip velocity at the boundary, the stresslet $\mathbf{S}$ is a traceless symmetric tensor invariant by rotation around $\mathbf{e}_{z}$ and must therefore be of the form $\mathbf{S}=S\left(\mathbf{e}_{z} \mathbf{e}_{z}-\frac{1}{3} \mathbf{I}\right)$. It is thus sufficient to use as dual velocity field the axisymmetric solution of Stokes's equations decaying at infinity and satisfying $\overline{\mathbf{u}}=E\left(\mathbf{e}_{z} \mathbf{e}_{z}-\frac{1}{3} \mathbf{I}\right) \cdot \mathbf{x}$ on the spheroid's boundary with arbitrary $E$. Following classical work [40], the dual velocity field $\overline{\mathbf{u}}$ and associated fluid force on the particle $\overline{\boldsymbol{\sigma}} \cdot \mathbf{n}$ can be found explicitly. In particular, we have

$$
\overline{\boldsymbol{\sigma}} \cdot \mathbf{n}=2 \mu\left[\frac{2 E G(\xi)}{9 F(\xi)} \mathbf{I}+\left(1-\frac{2}{3 F(\xi)}\right) \mathbf{E}\right] \cdot \mathbf{n},
$$

where $\xi \equiv a / b$ is the aspect ratio and the function $F$ is

$$
F(\xi)=\frac{1}{\left(\xi^{2}-1\right)^{2}}\left[-3 \xi^{2}+\frac{\xi\left(1+2 \xi^{2}\right)}{\sqrt{1-\xi^{2}}} \cos ^{-1} \xi\right],
$$

while the function $G$ is not required for what follows [35]. Using our integral formulations, one then easily obtains

$$
\mathbf{S}=-\frac{2 \mu}{3 F(\xi)} \int_{\partial V}\left(\mathbf{u}^{s} \mathbf{n}+\mathbf{n} \mathbf{u}^{s}\right) d A
$$

with $\mathbf{u}^{s}$ the prescribed slip velocity at the particle's boundary. This new result is valid for both prolate $(\xi \geq 1)$ and oblate $(\xi \leq 1)$ spheroids [note that $F(1)=4 / 15$, agreeing with Eq. (11)].

We use spheroidal polar coordinates $(\tau, \zeta, \phi)$ with $(x, y)=k \sqrt{\tau^{2} \mp 1} \sqrt{1-\zeta^{2}}(\cos \phi, \sin \phi)$ (for prolate and oblate spheroids, respectively), $z=k \zeta \tau, k=$ $\sqrt{\mathscr{S}\left|\xi^{2}-1\right| / 2 \pi H(\xi)}$, with $\mathscr{S}$, the surface area of the spheroid, and

$$
H(\xi)=1+\frac{\xi^{2}}{\sqrt{\xi^{2}-1}} \cos ^{-1}\left(\frac{1}{\xi}\right) .
$$

The surface of the particle is then defined by $\tau=\tau_{0}=\xi / \sqrt{\left|\xi^{2}-1\right|}$. For an active particle that prescribes an axisymmetric slip velocity $\mathbf{u}^{s}=u^{s}(\zeta) \mathbf{e}_{\zeta}$, the strength of the stresslet is then obtained as the integral

$$
S=-\frac{2 \mathscr{S} \mu}{F(\xi) H(\xi)} \int_{-1}^{1} u^{s}(\zeta) \zeta \sqrt{\frac{\xi^{2}\left(1-\zeta^{2}\right)}{\zeta^{2}+\xi^{2}\left(1-\zeta^{2}\right)}} d \zeta
$$

We can now apply this result to an autophoretic spheroidal particle releasing a solute of diffusivity $D$ with fixed flux $\mathcal{A}(\zeta)$ along its boundary. Interactions between the particle surface and the solute lead to a phoretic fluid slip velocity, $\mathbf{u}=\mathcal{M}(\zeta)(I-\mathbf{n n}) \cdot \nabla C$, induced along its boundary [41]. When solute advection is negligible, its concentration $C$ is the solution to the diffusive problem

$$
D \nabla^{2} C=0,\left.\quad D \mathbf{e}_{\tau} \cdot \nabla C\right|_{\partial V}=-\mathcal{A}(\zeta), \quad C(\infty)=0 .
$$

With the new integral result above, Eq. (22), we can now obtain the stresslet generated by the catalytic particle without solving the actual Stokes flow problem. Since Laplace's equation is separable in spheroidal coordinates, Eq. (23) can be solved explicitly for $c$ as 


$$
\begin{aligned}
& C(\tau, \zeta)=-\sum_{n=0}^{\infty} \frac{k(2 n+1) C_{n}(\tau)}{2 D C_{n}^{\prime}\left(\tau_{0}\right)} \mathcal{I}_{n}(\xi) L_{n}(\zeta), \\
& \mathcal{I}_{n}(\xi)=\int_{-1}^{1} \mathcal{A}(\zeta) \sqrt{\zeta^{2}+\xi^{2}\left(1-\zeta^{2}\right)} L_{n}(\zeta) d \zeta,
\end{aligned}
$$

where $C_{n}(\tau)=Q_{n}(\tau)$ or $Q_{n}(\mathrm{i} \tau)$ for prolate and oblate spheroids, respectively, and $L_{n}$ and $Q_{n}$ are the Legendre polynomials and function of the second kind, respectively. The general expression for the resulting stresslet of a spheroid, Eq. (22), can now be evaluated as $\mathbf{S}=$ $S\left(\mathbf{e}_{z} \mathbf{e}_{z}-\frac{1}{3} \mathbf{I}\right)$, with strength

$$
S=-\frac{\mu \xi}{D F(\xi)} \sqrt{\frac{8 \pi \mathscr{S}}{H(\xi)}} \int_{-1}^{1} \frac{\mathcal{M}(\zeta) \zeta\left(1-\zeta^{2}\right)}{\zeta^{2}+\xi^{2}\left(1-\zeta^{2}\right)} \frac{\partial c}{\partial \zeta} d \zeta
$$

Using Eq. (24), the stresslet intensity $S$ of a catalytic spheroidal particle of aspect ratio $\xi$ is finally obtained as

$$
S=\frac{\mu \mathscr{S}}{D} \frac{\xi \sqrt{\left|\xi^{2}-1\right|}}{F(\xi) H(\xi)} \sum_{n=1}^{\infty} \mathcal{I}_{n}(\xi) \mathcal{J}_{n}(\xi) \mathcal{K}_{n}(\xi),
$$

with

$$
\mathcal{J}_{n}(\xi)=\int_{-1}^{1} \frac{\zeta\left(1-\zeta^{2}\right) M(\zeta) L_{n}^{\prime}(\zeta)}{\zeta^{2}+\xi^{2}\left(1-\zeta^{2}\right)} d \zeta
$$

$$
\mathcal{K}_{n}(\xi)=\frac{(2 n+1) C_{n}\left(\frac{\xi}{\sqrt{\left|\xi^{2}-1\right|}}\right)}{C_{n}^{\prime}\left(\frac{\xi}{\sqrt{\left|\xi^{2}-1\right|}}\right)}
$$

This new result, impossible to compute directly analytically otherwise, allows one to characterize the role of geometry on the strength of the stresslet for active particles. For illustration, let us focus on a Janus particle with an active half $(\zeta>0)$ of uniform activity and mobility, and an inert half $(\zeta<0)$, where both quantities are zero. We plot in Fig. 1 the strength of the stresslet as a function of the aspect ratio of the Janus particle, showing the critical role of geometry. For positive activity (i.e., solute release on the surface of the particle) and positive mobility (i.e., slip velocity in the same direction as the local concentration gradient), oblate particles act as pushers $(S<0)$ while most prolate particles are pullers $(S>0)$. The spherical limit $(\xi=1)$ corresponds to a weak pusher swimmer while the pusher-puller transition occurs for a blunt prolate with aspect ratio $\xi \approx 2$ (Fig. 1)

These results can be rationalized physically by inspecting the distribution of solute around the particle (see Fig. 1, insets). For an oblate or spherical particle, the highest solute concentrations are found at the active pole. The slip velocity along the active boundaries is therefore oriented from the equator to the pole leading to a pusher-type signature on the flow. In contrast, for a prolate phoretic particle, the sharp local curvature near the active pole

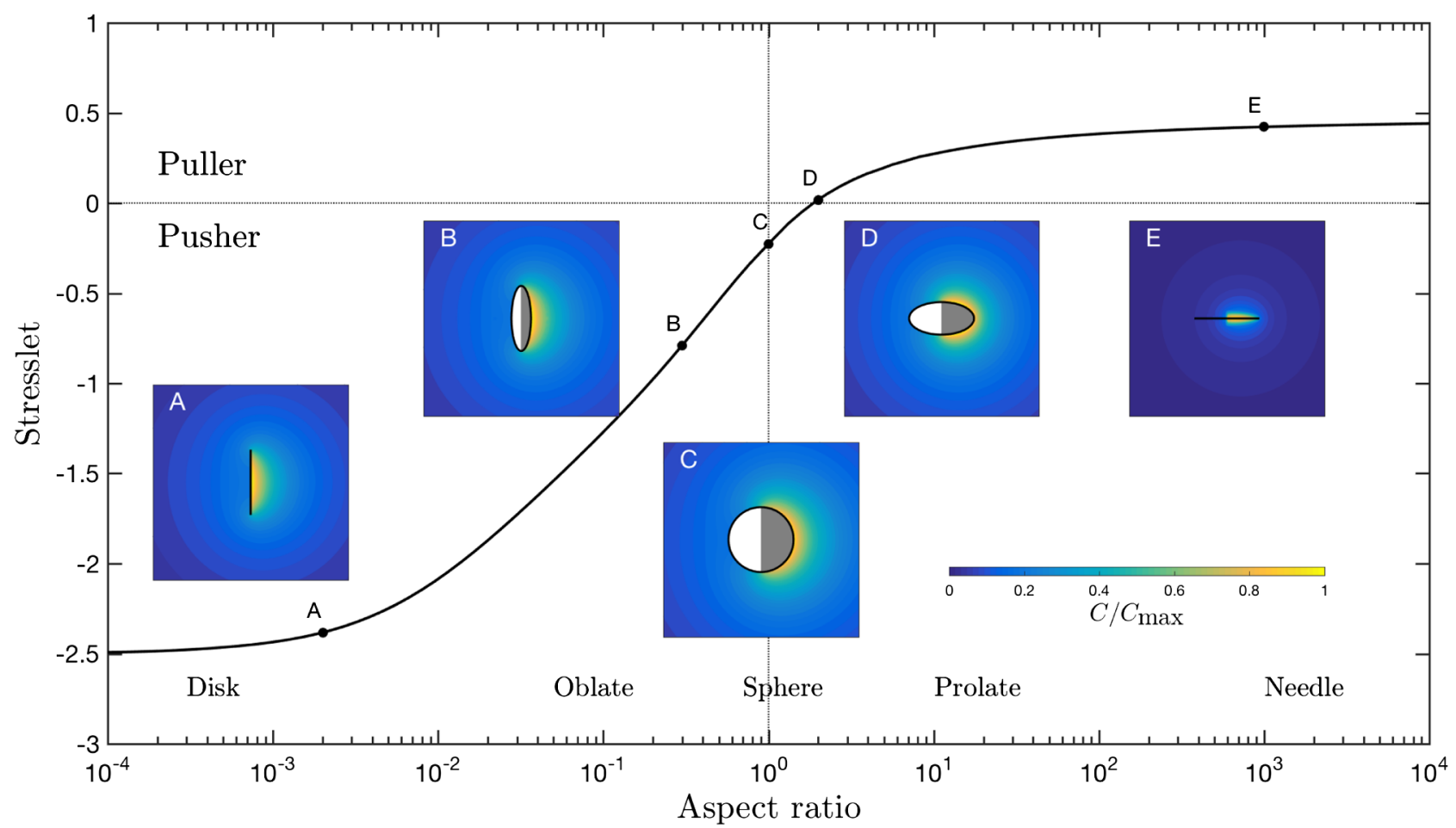

FIG. 1. Stresslet intensity (scaled by $\mu \mathscr{S} \mathcal{A}_{0} \mathcal{M}_{0} / D$ ) for a phoretic Janus particle of spheroidal shape as a function of its aspect ratio with $\mathcal{A}(\zeta)=\mathcal{A}_{0}$ and $\mathcal{M}(\zeta)=\mathcal{M}_{0}$ on the active right half (gray) and $\mathcal{A}(\zeta)=\mathcal{M}(\zeta)=0$ on the inert left half (white). The sign of stresslet is reversed by changing the sign of either $\mathcal{A}_{0}$ or $\mathcal{M}_{0}$, but not both. The distribution of concentration is also shown. 
results in a local minimum of the concentration at the pole (chemical solute is efficiently diffused away from that point) and the absolute maximum of the surface concentration is instead found at an intermediate position on the active half of the particle. When $\xi \rightarrow \infty$, one can show that this local maximum of concentration is found at a distance $z_{\max } \approx 0.2 a$ away from the equator. In that case, the slip velocity is still oriented from the equator to the pole for $0 \leq z \leq z_{\max }$ but in the reverse direction for $z_{\max } \leq z \leq a$, the latter being dominant and inducing a puller signature.

In summary, we outlined in this work a new method, based on the reciprocal theorem for Stokes flows, to compute the stresslet generated by an active particle. The method requires knowledge of (i) the instantaneous geometry of the particle, (ii) the prescribed slip velocity along its boundary, and (iii) a dual Stokes problem of an identical rigid particle in a linear flow. The main advantage of this approach is that it does not require one to solve for the actual flow field around the active particle. After the formal derivation of the method, we verified it for the classical cases of active spheres and rods for which an alternative, direct calculation is possible [35]. We then demonstrated how to use our new integral formulation to derive a result impossible to obtain directly, namely, the stresslet for spheroidal phoretic particles.

As an extension for future work, we note that when the particle is not torque free, the present approach could easily be generalized to compute the rotlet generated by the active particle (i.e., the strength of the torque locally induced by the swimmer) by repeating the analysis presented in this Letter with a dual flow field where the second-rank tensor $\mathbf{E}$ is antisymmetric.

We envision our method to be particularly relevant to fixed-shape phoretic swimmers where the dual problem can be solved once and for all. The result of Eq. (27) could then be directly used to sculpt the strength of the stresslet as a function of the chemical and geometrical characteristics of the particle, allowing one to potentially tune interactions of active particles with boundaries and to create active fluids with predesigned collective or rheological characteristics.

Funding from the EU (CIG to E. L.) and by the French Ministry of Defense (DGA to S. M.) is gratefully acknowledged.

*e.lauga@damtp.cam.ac.uk

† sebastien.michelin@ladhyx.polytechnique.fr

[1] J. Lighthill, SIAM Rev. 18, 161 (1976).

[2] E. Lauga and T. R. Powers, Rep. Prog. Phys. 72, 096601 (2009).

[3] E. Lauga, Annu. Rev. Fluid Mech. 48, 105 (2016).

[4] L. J. Fauci and R. Dillon, Annu. Rev. Fluid Mech. 38, 371 (2006).

[5] J. S. Guasto, R. Rusconi, and R. Stocker, Annu. Rev. Fluid Mech. 44, 373 (2012).

[6] D. L. Koch and G. Subramanian, Annu. Rev. Fluid Mech. 43, 637 (2011).
[7] H. C. Berg, E. coli in Motion (Springer-Verlag, New York, 2004).

[8] W. F. Paxton, S. Sundararajan, T. E. Mallouk, and A. Sen, Angew. Chem. 45, 5420 (2006).

[9] R. Golestanian, T. B. Liverpool, and A. Ajdari, New J. Phys. 9, 126 (2007).

[10] S. Ramaswamy, Annu. Rev. Condens. Matter Phys. 1, 323 (2010).

[11] M. Marchetti, J. Joanny, S. Ramaswamy, T. Liverpool, J. Prost, M. Rao, and R. A. Simha, Rev. Mod. Phys. 85, 1143 (2013).

[12] C. Brennen and H. Winet, Annu. Rev. Fluid Mech. 9, 339 (1977).

[13] R. G. Cox, J. Fluid Mech. 44, 791 (1970).

[14] R. E. Johnson, J. Fluid Mech. 99, 411 (1980).

[15] H. C. Berg, Random Walks in Biology (Princeton University Press, Princeton, NJ, 1993).

[16] G. K. Batchelor, J. Fluid Mech. 41, 545 (1970).

[17] D. C. Guell, H. Brenner, R. B. Frankel, and H. Hartman, J. Theor. Biol. 135, 525 (1988).

[18] D. Saintillan and M. J. Shelley, C.R. Phys. 14, 497 (2013).

[19] C. Dombrowski, L. Cisneros, S. Chatkaew, R. E. Goldstein, and J. O. Kessler, Phys. Rev. Lett. 93, 098103 (2004).

[20] A. Sokolov, I. S. Aranson, J. O. Kessler, and R. E. Goldstein, Phys. Rev. Lett. 98, 158102 (2007).

[21] A. P. Berke, L. Turner, H. C. Berg, and E. Lauga, Phys. Rev. Lett. 101, 038102 (2008).

[22] K. Drescher, K. C. Leptos, I. Tuval, T. Ishikawa, T. J. Pedley, and R. E. Goldstein, Phys. Rev. Lett. 102, 168101 (2009).

[23] X. L. Wu and A. Libchaber, Phys. Rev. Lett. 84, 3017 (2000).

[24] A. Jepson, V. A. Martinez, J. Schwarz-Linek, A. Morozov, and W. C. K. Poon, Phys. Rev. E 88, 041002 (2013).

[25] D. Saintillan, J. Exp. Mech. 50, 1275 (2010).

[26] T. Ishikawa, G. Sekiya, Y. Imai, and T. Yamaguchi, Biophys. J. 93, 2217 (2007).

[27] K. Drescher, J. Dunkel, L. H. Cisneros, S. Ganguly, and R. E. Goldstein, Proc. Natl. Acad. Sci. U.S.A. 108, 10940 (2011).

[28] H. A. Stone and A. D. T. Samuel, Phys. Rev. Lett. 77, 4102 (1996).

[29] L. G. Leal, Annu. Rev. Fluid Mech. 12, 435 (1980).

[30] R. V. Raja, G. Subramanian, and D. L. Koch, J. Fluid Mech. 646, 255 (2010).

[31] O. S. Pak, J. Feng, and H. A. Stone, J. Fluid Mech. 753, 535 (2014).

[32] S. Michelin and E. Lauga, Phys. Fluids 27, 111701 (2015).

[33] L. G. Leal, Advanced Transport Phenomena: Fluid Mechanics and Convective Transport Processes (Cambridge University Press, Cambridge, England, 2007).

[34] J. R. Blake, J. Fluid Mech. 46, 199 (1971).

[35] See Supplemental Material at http://link.aps.org/ supplemental/10.1103/PhysRevLett.117.148001, which includes Ref. [36], for details of the direct calculation of stresslets in the cases of a spherical squirmer, an active rod and an ellipsoidal particle.

[36] H. Lamb, Hydrodynamics, 6th ed. (Dover, New York, 1932).

[37] S. Michelin and E. Lauga, J. Fluid Mech. 747, 572 (2014).

[38] O. S. Pak and E. Lauga, J. Eng. Math. 88, 1 (2014).

[39] A. Kanevsky, M. J. Shelley, and A.-K. Tornberg, J. Comput. Phys. 229, 958 (2010).

[40] G. B. Jeffery, Proc. R. Soc. A 102, 161 (1922).

[41] J. Anderson, Annu. Rev. Fluid Mech. 21, 61 (1989). 


\title{
Supplemental Information to "Stresslets induced by active swimmers"
}

\author{
Eric Lauga ${ }^{1, *}$ and Sebastien Michelin ${ }^{2, \dagger}$ \\ ${ }^{1}$ Department of Applied Mathematics and Theoretical Physics, \\ University of Cambridge, CB3 OWA, United Kingdom. \\ ${ }^{2}$ LadHyX - Département de Mécanique, Ecole Polytechnique - CNRS, 91128 Palaiseau, France.
}

(Dated: September 9, 2016)

\section{DIRECT CALCULATION OF THE STRESSLET OF A SQUIRMING SPHERE}

In the reference frame of a translating force-free sphere, the velocity field generated by an axisymmetric tangential slip flow at the sphere's boundary $\mathbf{u}(r=a)=u_{s}(\zeta) \mathbf{e}_{\theta}$ can be written as a superposition of squirming modes [1,2]. With $\zeta=\cos \theta$ in spherical polar coordinates,

$$
\mathbf{u}=\sum_{n=1}^{\infty} \frac{(2 n+1) \alpha_{n}}{n(n+1)}\left[\frac{\psi_{n}(r)}{r^{2}} L_{n}(\zeta) \mathbf{e}_{r}-\sqrt{1-\zeta^{2}} L_{n}^{\prime}(\zeta) \frac{\psi_{n}^{\prime}(r)}{r} \mathbf{e}_{\theta}\right]
$$

with

$$
\begin{aligned}
& \psi_{1}(r)=\frac{a^{3}-r^{3}}{3 r}, \quad \psi_{n \geq 2}(r)=\frac{1}{2}\left(\frac{a^{n}}{r^{n-2}}-\frac{a^{n+2}}{r^{n}}\right), \\
& \alpha_{n}(t)=\frac{1}{2} \int_{-1}^{1} u_{s}(\zeta) \sqrt{1-\zeta^{2}} L_{n}^{\prime}(\zeta) \mathrm{d} \zeta .
\end{aligned}
$$

The traction on the boundary of the squirmer $(r=a)$ can also be computed explicitly as

$$
\boldsymbol{\sigma} \cdot \mathbf{n}=-\frac{3 \mu \alpha_{1}}{a}\left(2 \zeta \mathbf{e}_{r}+\sqrt{1-\zeta^{2}} \mathbf{e}_{\theta}\right)-\frac{\mu}{a} \sum_{n=2}^{\infty} \frac{(2 n+1) \alpha_{n}}{n(n+1)}\left[3 n L_{n}(\zeta) \mathbf{e}_{r}+(2 n+1) \sqrt{1-\zeta^{2}} L_{n}^{\prime}(\zeta) \mathbf{e}_{\theta}\right] .
$$

Using the definition of the stresslet, Eq. (1) in the main article, with $\mathbf{n}=\mathbf{e}_{r}$ and $\mathbf{x}=a \mathbf{e}_{r}$ on the boundary

$$
\begin{aligned}
\mathbf{S} & =2 \pi \mu a^{2} \int_{-1}^{1}\left[a \sigma_{r r}\left\langle\mathbf{e}_{r} \mathbf{e}_{r}\right\rangle_{\phi}+\left(a \sigma_{r \theta}-2 \mu u_{\theta}\right) \frac{\left\langle\mathbf{e}_{r} \mathbf{e}_{\theta}\right\rangle_{\phi}+\left\langle\mathbf{e}_{\theta} \mathbf{e}_{r}\right\rangle_{\phi}}{2}\right] \mathrm{d} \zeta \\
& =2 \pi \mu a^{2}\left(\mathbf{e}_{z} \mathbf{e}_{z}-\frac{\mathbf{I}}{3}\right) \int_{-1}^{1}\left[a \sigma_{r r} L_{2}(\zeta)-\frac{1}{2}\left(a \sigma_{r \theta}-2 \mu u_{\theta}\right) L_{2}^{\prime}(\zeta) \sqrt{1-\zeta^{2}}\right] \mathrm{d} \zeta \\
& =10 \pi a^{2} \mu \alpha_{2}\left(\mathbf{e}_{z} \mathbf{e}_{z}-\frac{\mathbf{I}}{3}\right)
\end{aligned}
$$

or finally

$$
\mathbf{S}=15 \pi a^{2} \mu\left(\mathbf{e}_{z} \mathbf{e}_{z}-\frac{\mathbf{I}}{3}\right) \int_{-1}^{1} \zeta \sqrt{1-\zeta^{2}} u_{s}(\zeta) \mathrm{d} \zeta
$$

\section{RECIPROCAL CALCULATION OF THE STRESSLET OF A SQUIRMING SPHERE}

Using the results presented in this article, the stresslet of the squirming sphere can be directly computed from the slip velocity distribution provided one is able to find the tensor $\boldsymbol{\Sigma}$ such that the perturbation to the stress field

\footnotetext{
*Electronic address: e.lauga@damtp.cam.ac.uk

${ }^{\dagger}$ Electronic address: sebastien.michelin@ladhyx.polytechnique.fr
} 
introduced by a fixed rigid particle in a linear flow field $\mathbf{u}=-\mathbf{E} \cdot \mathbf{x}$ is defined as $\overline{\boldsymbol{\sigma}}=\boldsymbol{\Sigma}: \mathbf{E}$. For a given $\mathbf{E}$, the perturbation flow and pressure field are obtained [3] as

$$
\begin{aligned}
\overline{\mathbf{u}} & =a^{5} \frac{\mathbf{E} \cdot \mathbf{x}}{r^{5}}+\frac{5(\mathbf{x} \cdot \mathbf{E} \cdot \mathbf{x}) \mathbf{x}}{2}\left(\frac{a^{3}}{r^{5}}-\frac{a^{5}}{r^{7}}\right), \\
\bar{p} & =5 a^{3} \mu \frac{\mathbf{x} \cdot \mathbf{E} \cdot \mathbf{x}}{r^{5}}
\end{aligned}
$$

Taking the symmetric part of the gradient of the flow field, the stress tensor $\boldsymbol{\sigma}=-\bar{p} \mathbf{1}+\mu\left(\nabla \overline{\mathbf{u}}+\nabla \overline{\mathbf{u}}^{T}\right)$ is computed as

$\overline{\boldsymbol{\sigma}}=\frac{2 \mu a^{5}}{r^{5}} \mathbf{E}+\frac{5 \mu a^{3}}{r^{5}}(\mathbf{x}(\mathbf{E} \cdot \mathbf{x})+(\mathbf{E} \cdot \mathbf{x}) \mathbf{x})-\frac{5 \mu a^{5}}{r^{7}}((\mathbf{x} \cdot \mathbf{E} \cdot \mathbf{x}) \mathbf{I}+2 \mathbf{x}(\mathbf{E} \cdot \mathbf{x})+2(\mathbf{E} \cdot \mathbf{x}) \mathbf{x})+5 \mu(\mathbf{x} \cdot \mathbf{E} \cdot \mathbf{x}) \mathbf{x} \mathbf{x}\left(\frac{7 a^{5}}{r^{9}}-\frac{5 a^{3}}{r^{7}}\right)$

Therefore, from the definition of $\boldsymbol{\Sigma}$,

$$
\begin{aligned}
\Sigma_{k l i j} & =\frac{\mu a^{5}}{r^{5}}\left(\delta_{i k} \delta_{j l}+\delta_{j k} \delta_{i l}\right)+\frac{5 \mu a^{3}}{2 r^{5}}\left(\delta_{i l} x_{j} x_{k}+\delta_{k i} x_{j} x_{l}+\delta_{j l} x_{i} x_{k}+\delta_{k j} x_{i} x_{l}\right) \\
& -\frac{5 \mu a^{5}}{r^{7}}\left(\delta_{k l} x_{i} x_{j}+\delta_{j l} x_{k} x_{i}+\delta_{i l} x_{j} x_{k}+\delta_{j k} x_{i} x_{l}+\delta_{i k} x_{j} x_{l}\right)+5 \mu\left(\frac{7 a^{5}}{r^{9}}-\frac{5 a^{3}}{r^{7}}\right) x_{i} x_{j} x_{k} x_{l},
\end{aligned}
$$

and on the sphere's surface

$$
\Sigma_{k l i j}-\frac{1}{3} \Sigma_{k l m m} \delta_{i j}-\mu \delta_{i k} \delta_{j l}-\mu \delta_{i l} \delta_{j k}=-\frac{5}{2} \mu\left(\delta_{i l} n_{j} n_{k}+\delta_{i k} n_{j} n_{l}+\delta_{j l} n_{i} n_{k}+\delta_{j k} n_{i} n_{l}+2 \delta_{k l} n_{i} n_{j}-4 n_{i} n_{j} n_{k} n_{l}\right)+\mu \delta_{k l} \delta_{i j} .
$$

Then, applying the fundamental result of the main article, Eq. (7), the stresslet of a general spherical active particle is obtained as

$$
\begin{aligned}
S_{i j} & =\mu \iint_{\partial V} n_{l} u_{k}\left[-\frac{5}{2}\left(\delta_{i l} n_{j} n_{k}+\delta_{i k} n_{j} n_{l}+\delta_{j l} n_{i} n_{k}+\delta_{j k} n_{i} n_{l}+2 \delta_{k l} n_{i} n_{j}-4 n_{i} n_{j} n_{k} n_{l}\right)+\delta_{k l} \delta_{i j}\right] \mathrm{d} A \\
& =-\frac{5 \mu}{2} \iint_{\partial V}\left(n_{i} u_{j}+n_{j} u_{i}\right) \mathrm{d} A .
\end{aligned}
$$

\section{DIRECT CALCULATION OF THE STRESSLET OF AN ACTIVE ROD}

An active rod of length $L$ and orientation vector $\mathbf{p}$ is considered. The rod is slender enough so that resistive force theory can be used to determine the force density applied on the rod. First we note that the last terms in Eq. (1) of the main article disappear since

$$
\iint_{\partial V} u_{i} n_{j} \mathrm{~d} A=\int_{L} u(s) \mathbf{p}_{i}\left(\int_{\partial V_{R}(s)} n_{j} \mathrm{~d} A_{R}(s)\right) \mathrm{d} s
$$

where $A_{R}(s)$ is the circle around the rod at section $s$. Clearly by symmetry the integral $\int_{\partial V_{R}(s)} \mathbf{n d} A_{R}(s)=\mathbf{0}$ and thus these terms disappear. Calling $\mathbf{f}$ the force per unit length exerted on the swimming rod, the stresslet is given by

$$
\mathbf{S}=\int_{L}\left[\frac{1}{2}(\mathbf{f} \mathbf{x}+\mathbf{x f})-\frac{1}{3}(\mathbf{f} \cdot \mathbf{x}) \mathbf{I}\right] \mathrm{d} s
$$

To determine $\mathbf{f}$ we need to pay a closer look to the velocity distribution on the rod. We call $U \mathbf{p}$ the swimming speed and write the swimming gait as $u(s)=U_{0} \alpha(s)$ where $U_{0}$ is a characteristic velocity and $\alpha$ a dimensionless function characterising the distribution of velocity. The total velocity relative to the fluid at rest at infinity is

$$
\mathbf{u}=U_{0} \alpha(s) \mathbf{p}+U \mathbf{p},
$$

resulting (resistive force theory) into a force per unit length $\mathbf{f}$ given by

$$
\mathbf{f}=-\zeta_{\|}\left[U_{0} \alpha(s)+U\right] \mathbf{p},
$$


where $\zeta_{\|}$is the local tangential drag coefficient along the rod. Inertia is negligible and the rod is force-free, therefore $\int \mathbf{f} \mathrm{d} s=\mathbf{0}$ and thus the swimming speed is given by

$$
U=-U_{0}\langle\alpha\rangle
$$

where $\langle\alpha\rangle=\left(\int_{L} \alpha(s) \mathrm{d} s\right) / L$. The resulting force density is thus

$$
\mathbf{f}(s)=-\zeta_{\|} U_{0}[\alpha(s)-\langle\alpha\rangle] \mathbf{p} .
$$

From this result and using $\mathbf{x}=s \mathbf{p}$ and $\zeta_{\|}=\zeta_{\perp} / 2$ with $\zeta_{\perp}$ the normal drag coefficient, the stresslet can be computed from Eq. (14) above:

$$
\mathbf{S}=-\zeta_{\|} \int_{L}\left(U_{0} s[\alpha(s)-\langle\alpha\rangle] \mathbf{p} \mathbf{p}-\frac{1}{3} U_{0} s[\alpha(s)-\langle\alpha\rangle] \mathbf{I}\right) \mathrm{d} s=-\frac{\zeta_{\perp}}{2}\left(U_{0} \int_{L} s[\alpha(s)-\langle\alpha\rangle] \mathrm{d} s\right)\left(\mathbf{p} \mathbf{p}-\frac{1}{3} \mathbf{I}\right)
$$

Defining $s$ in $[-L / 2, L / 2]$, this result is strictly equivalent to the reciprocal calculation presented in the main text, Eq. (17).

\section{ELLIPSOID}

\section{A. Spheroidal coordinates}

We consider here a general axisymmetric ellipsoid of axis $\mathbf{e}_{z}$, and note $a$ (resp. $b$ ) the semi-axis along $\mathbf{e}_{z}$ (in the plane normal to $\mathbf{e}_{z}$ ). The ellispoid is characterized by its surface are noted $\mathscr{S}$ and its aspect ratio $\xi=a / b . \xi \geq 1$ (resp. $\xi \leq 1$ ) corresponds to a prolate (resp. oblate) spheroid. Prolate (resp. oblate) spheroidal coordinates $(\tau, \zeta, \phi)$ are defined such that $z=k \tau \zeta$ and $(x, y)=k \sqrt{\left(\tau^{2}-1\right)\left(1-\zeta^{2}\right)}(\cos \phi, \sin \phi)\left(\right.$ resp. $\left.(x, y)=k \sqrt{\left(\tau^{2}+1\right)\left(1-\zeta^{2}\right)}(\cos \phi, \sin \phi)\right)$. $k$ is a positive scaling constant that relates the aspect ratio $\xi$ and the surface area of the ellipsoid (see main text). The unit vectors and scale factors can be expressed for both oblate and prolate spheroids, at the surface of the ellispoid only (i.e. for $\tau=\xi / \sqrt{\left|\xi^{2}-1\right|}$ ), as

$$
\begin{gathered}
h_{\tau}=k \sqrt{\zeta^{2}+\xi^{2}\left(1-\zeta^{2}\right)}, \quad \mathbf{e}_{\tau}=\frac{\xi \sqrt{1-\zeta^{2}}\left(\mathbf{e}_{\phi} \times \mathbf{e}_{z}\right)+\zeta \mathbf{e}_{z}}{\sqrt{\zeta^{2}+\xi^{2}\left(1-\zeta^{2}\right)}} \\
h_{\zeta}=k \sqrt{\frac{\zeta^{2}+\xi^{2}\left(1-\zeta^{2}\right)}{\left(1-\zeta^{2}\right)\left|\xi^{2}-1\right|}}, \quad \mathbf{e}_{\zeta}=\frac{-\zeta\left(\mathbf{e}_{\phi} \times \mathbf{e}_{z}\right)+\xi \sqrt{1-\zeta^{2}} \mathbf{e}_{z}}{\sqrt{\zeta^{2}+\xi^{2}\left(1-\zeta^{2}\right)}} \\
h_{\phi}=k \sqrt{\frac{1-\zeta^{2}}{\left|\xi^{2}-1\right|}}, \quad \mathbf{e}_{\phi}=-\sin \phi \mathbf{e}_{x}+\cos \phi \mathbf{e}_{y} .
\end{gathered}
$$

The area of the ellipsoid's surface can be expressed as

$$
\mathscr{S}=\frac{2 \pi k^{2}}{\left|\xi^{2}-1\right|}\left[1+\frac{\xi^{2}}{\sqrt{\xi^{2}-1}} \cos ^{-1}\left(\frac{1}{\xi}\right)\right]
$$

\section{B. Jeffery's solution}

Following [4], the solution of Stokes' equations that decay at infinity and satisfies $\overline{\mathbf{u}}=E\left(\mathbf{e}_{z} \mathbf{e}_{z}-\mathbf{1} / 3\right) \cdot \mathbf{x}$ at the spheroid's boundary is obtained as

$$
\overline{\mathbf{u}}=\nabla \times(\mathbf{B} \cdot \chi)+[(\mathbf{A} \cdot \mathbf{x}) \cdot \nabla] \nabla \varphi-\mathbf{A} \cdot \nabla \varphi,
$$


with

$$
\begin{aligned}
\varphi(\mathbf{x}) & =\int_{\lambda(\mathbf{x})}^{\infty}\left[\frac{z^{2}}{a^{2}+u}+\frac{x^{2}+y^{2}}{b^{2}+u}\right] \frac{\mathrm{d} u}{\left(b^{2}+u\right) \sqrt{a^{2}+u}}, \\
\chi(\mathbf{x}) & =\left(x z \mathbf{e}_{y}+y z \mathbf{e}_{x}\right) \int_{\lambda(\mathbf{x})}^{\infty} \frac{\mathrm{d} u}{\left(a^{2}+u\right)^{3 / 2}\left(b^{2}+u\right)^{2}}, \\
\mathbf{A} & =-\frac{b^{3}}{6} \frac{\mathbf{E}}{F(\xi)}, \quad F(\xi)=\xi \int_{0}^{\infty} \frac{u \mathrm{~d} u}{(1+u)^{2}\left(\xi^{2}+u\right)^{3 / 2}}, \\
\mathbf{B} & =-\frac{b^{3}\left(2 a^{2}+b^{2}\right) E}{9 F(\xi)}\left(-\mathbf{e}_{x} \mathbf{e}_{x}+\mathbf{e}_{y} \mathbf{e}_{y}\right),
\end{aligned}
$$

and $\lambda(\mathbf{x})$ is the positive root of

$$
\frac{z^{2}}{a^{2}+\lambda}+\frac{x^{2}+y^{2}}{b^{2}+\lambda}=1
$$

The force applied on the spheroid's boundary is then

$$
\overline{\boldsymbol{\sigma}} \cdot \mathbf{n}=2 \mu\left[\frac{2 G(\xi)}{9 F(\xi)} E \mathbf{1}+\left(1-\frac{2}{3 F(\xi)}\right) \mathbf{E}\right] \cdot \mathbf{n},
$$

with

$$
G(\xi)=\int_{0}^{\infty} \frac{\xi\left(1-\xi^{2}\right) \mathrm{d} u}{(1+u)^{2}\left(\xi^{2}+u\right)^{3 / 2}}
$$

\section{Reciprocal calculation of the stresslet of an active ellipsoid}

Considering now an active spheroid prescribing a slip velocity at its boundary, the approach followed to derive Eq. (6) in the main text can be adapted to the present axisymmetric setting in order to derive the traction part of the resulting stresslet as

$$
\iint_{\partial V} \frac{1}{2}\left[(\mathbf{x}(\boldsymbol{\sigma} \cdot \mathbf{n})+(\boldsymbol{\sigma} \cdot \mathbf{n}) \mathbf{x})-\frac{\mathbf{x} \cdot \boldsymbol{\sigma} \cdot \mathbf{n}}{3} \mathbf{1}\right] \mathrm{d} S=\mu\left(1-\frac{2}{3 F(\xi)}\right) \iint_{\partial V}(\mathbf{u n}+\mathbf{n u}) \mathrm{d} S,
$$

and finally

$$
\mathbf{S}=-\frac{2 \mu}{3 F(\xi)} \iint_{\partial V}(\mathbf{u n}+\mathbf{n u}) \mathrm{d} S
$$

\section{Finding the concentration distribution around an axisymmetric catalytic particle}

The previous result can be used to determine the stresslet generated by an active autophoretic (or catalytic) particle of spheroidal shape whose surface properties are axisymmetric and characterized by a chemical activity (i.e. solute release rate) $\mathcal{A}(\zeta)$ and mobility $\mathcal{M}(\zeta)$. Writing $c$ the concentration of the solute, and neglecting advection, the solute dynamics is completely described by the following Laplace problem:

$$
D \nabla^{2} c=0,\left.\quad D \mathbf{e}_{\tau} \cdot \nabla c\right|_{\partial V}=-\mathcal{A}(\zeta), \quad c(\mathbf{x} \rightarrow \infty)=0,
$$

and the slip velocity imposed by the particle at its surface is computed as $\mathbf{u}_{s}=\mathcal{M}(\zeta)(\mathbf{1}-\mathbf{n n}) \cdot \nabla c$.

Laplace's equation is separable in spheroidal polar coordinates:

$$
\nabla^{2} c=\frac{1}{k^{2}\left(\tau^{2} \pm \zeta^{2}\right)}\left\{\frac{\partial}{\partial \tau}\left[\left(\tau^{2} \pm 1\right) \frac{\partial c}{\partial \tau}\right]+\frac{\partial}{\partial \zeta}\left[\left(1-\zeta^{2}\right) \frac{\partial c}{\partial \zeta}\right]\right\}
$$

where \pm corresponds to oblate and prolate spheroids, respectively. Its general solution is of the form

$$
c=\sum_{n=0}^{\infty} c_{n} C_{n}(\tau) L_{n}(\zeta)
$$


with $C_{n}(\tau)=Q_{n}(\tau)$ or $Q_{n}(\mathrm{i} \tau)$ for prolate and oblate spheroids, respectively, and $Q_{n}$ is the $n$-th Legendre function of second kind. Using $\mathbf{e}_{\tau} \cdot \nabla c=1 / h_{\tau}(\partial c / \partial \tau)$, the Neuman boundary condition for $c$ on the particle's surface can be projected along $L_{n}(\zeta)$ using the orthogonality of Legendre polynomials

$$
\int_{-1}^{1} L_{n}(\zeta) L_{p}(\zeta) \mathrm{d} \zeta=\frac{2 \delta_{n p}}{2 n+1}
$$

to obtain the concentration distribution

$$
\begin{aligned}
& c(\tau, \zeta)=-\sum_{n=0}^{\infty} \frac{k(2 n+1) C_{n}(\tau)}{2 D C_{n}^{\prime}\left(\tau_{0}\right)} \mathcal{I}_{n}(\xi) L_{n}(\zeta) \\
& \mathcal{I}_{n}(\xi)=\int_{-1}^{1} \mathcal{A}(\zeta) \sqrt{\zeta^{2}+\xi^{2}\left(1-\zeta^{2}\right)} L_{n}(\zeta) \mathrm{d} \zeta
\end{aligned}
$$

[1] H. Lamb. Hydrodynamics. Dover, New York, 6th edition, 1932.

[2] J. R. Blake. A spherical envelope approach to ciliary propulsion. J. Fluid Mech., 46:199-208, 1971.

[3] L. G. Leal. Advanced Transport Phenomena: Fluid Mechanics and Convective Transport Processes. Cambridge University Press, Cambridge, UK, 2007.

[4] G. B. Jeffery. The motion of ellipsoidal particles immersed in a viscous fluid. Proc. R. Soc. Lond. A, 102:161-179, 1922. 